bioRxiv preprint doi: https://doi.org/10.1101/2020.06.05.135913; this version posted October 31, 2020. The copyright holder for this preprint (which was not certified by peer review) is the author/funder, who has granted bioRxiv a license to display the preprint in perpetuity. It is made available under aCC-BY-NC-ND 4.0 International license.

\title{
Frequent loss-of-heterozygosity in CRISPR-Cas9-edited early human embryos
}

Gregorio Alanis-Lobatoa, Jasmin Zohren ${ }^{b}$, Afshan McCarthya, Norah M.E. Fogartya,c, Nada Kubikovad, Emily Hardmana, Maria Grecoe, Dagan Wells d.f, James M.A. Turnerb, Kathy K. Niakana,g,*

aHuman Embryo and Stem Cell Laboratory, The Francis Crick Institute, 1 Midland Road, London NW1 1AT, UK

bSex Chromosome Biology Laboratory, The Francis Crick Institute, 1 Midland Road, London NW1 1AT, UK cCentre for Stem Cells and Regenerative Medicine, King's College London, Guy's Campus, Great Maze Pond, London SE1 9RT, UK

dUniversity of Oxford, Winchester House, Heatley Road, Oxford Science Park, Oxford OX4 4GE, UK eAncient Genomics Laboratory, The Francis Crick Institute, 1 Midland Road, London NW1 1AT, UK fJuno Genetics, Winchester House, Heatley Road, Oxford Science Park, Oxford OX4 4GE, UK 9The Centre for Trophoblast Research, Department of Physiology, Development and Neuroscience, University of Cambridge, Cambridge CB2 3EG, UK

To whom correspondence should be addressed:

Kathy K. Niakan

Human Embryo and Stem Cell Laboratory

The Francis Crick Institute

1 Midland Road

London NW1 1AT

UK

kathy.niakan@crick.ac.uk

Keywords: genome editing, CRISPR-Cas9, human embryo, segmental aneuploidy, loss-of-heterozygosity 


\section{Abstract}

CRISPR-Cas9 genome editing is a promising technique for clinical applications, such as the correction of disease-associated alleles in somatic cells. The use of this approach has also been discussed in the context of heritable editing of the human germline. However, studies assessing gene correction in early human embryos report low efficiency of mutation repair, high rates of mosaicism and the possibility of unintended editing outcomes that may have pathologic consequences. We developed computational pipelines to assess single-cell genomics and transcriptomics datasets from OCT4 (POU5F1) CRISPR-Cas9-targeted and control human preimplantation embryos. This allowed us to evaluate on-target mutations that would be missed by more conventional genotyping techniques. We observed loss-of-heterozygosity in edited cells that spanned regions beyond the POU5F1 on-target locus, as well as segmental loss and gain of chromosome 6 , on which the POU5F1 gene is located. Unintended genome editing outcomes were present in approximately $16 \%$ of the human embryo cells analysed and spanned 4 to $20 \mathrm{~kb}$. Our observations are consistent with recent findings indicating complexity at on-target sites following CRISPR-Cas9 genome editing. Our work underscores the importance of further basic research to assess the safety of genome editing techniques in human embryos, which will inform debates about the potential clinical use of this technology.

\section{Introduction}

Clustered regularly interspaced short palindromic repeat (CRISPR)-CRISPR associated 9 (Cas9) genome editing is not only an indispensable molecular biology technique (1) but also has enormous therapeutic potential as a tool to correct disease-causing mutations (2). Genome editing of human embryos or germ cells to produce heritable changes has the potential to reduce the burden of genetic disease and its use in this context is currently a topic of international discussions centred around ethics, safety and efficiency $(3,4)$.

Several groups have conducted studies to assess the feasibility of gene correction in early human embryos (5-7) and they all encountered low efficiency of gene repair and high levels of mosaicism (i.e. embryos with corrected as well as mutant uncorrected blastomeres or blastomeres with unintended insertion/deletion mutations), which are unacceptable outcomes for clinical applications. In 2017, Ma et al. set out to correct a $4 \mathrm{bp}$ pathogenic heterozygous deletion in the MYBPC3 gene using the CRISPR-Cas9 system (8). The experimental strategy involved co-injection of Cas9 protein, a single guide RNA (sgRNA) that specifically targeted the MYBPC3 mutation and a repair template into either fertilised eggs (zygotes) or oocytes, coincident with intracytoplasmic sperm injection. Analysis of the resulting embryos revealed a higher than expected incidence, with respect to controls, of samples where only wild-type copies of the gene were detectable (8). Intriguingly, the excess of apparently uniformly homozygous wild-type embryos in both cases was not associated with use of the provided repair template for gene correction. Instead, the authors suggest that in edited embryos the wild-type maternal allele served as a template for the high-fidelity homology directed repair (HDR) pathway to repair the double-strand lesion caused by the Cas9 protein in the paternal allele (8).

Ma and colleagues' interpretation of gene editing by inter-homologue homologous recombination (IH-HR) in the early human embryo has been met with scepticism because alternative explanations can account for the observed results (9-11). One of these is that the CRISPR-Cas9 system can induce large deletions and complex genomic rearrangements with pathogenic potential at the on-target site $(9,10,12-14)$. These events can be overlooked because genotyping of the targeted genomic locus often involves the amplification of a small PCR fragment centred around the on-target cut-site. CRISPR-Cas9-induced deletions larger than these fragments in either direction would eliminate one or both PCR primer annealing sites. This in turn can lead to amplification of only one allele, giving the false impression that targeting was unsuccessful or that there is a single homozygous event at the on-target site $(9,10,15)$. Loss-of-heterozygosity $(\mathrm{LOH})$ can also be the result of more complex genomic rearrangements like inversions, large insertions, translocations, chromosome loss and even IH-HR with crossover, whereby a large piece of one parental allele is integrated by the other parental chromosome at the on-target cut-site (15).

The reported frequencies of unintended CRISPR-Cas9 on-target damage are not negligible. Adikusama et al. targeted six genes in a total of 127 early mouse embryos and detected large deletions (between 100bp and $2.3 \mathrm{~kb}$ ) in $45 \%$ of their samples using long-range PCR (10). Of note, large deletions were generally more prevalent when they targeted intronic regions $(>70 \%)$ than when they targeted exons $(20 \%)$. Consistent with this, Kosicki and colleagues observed large deletions (up to $6 \mathrm{~kb}$ ) and other complex genomic lesions at frequencies of $5-20 \%$ of their clones after targeting the PigA and Cd9 loci in two mouse embryonic stem cell (mESC) lines and primary mouse cells from the bone marrow, as well as the PIGA gene in immortalised human 
female retinal pigment epithelial cells (12). Moreover, Owens et al. used CRISPR-Cas9 with two sgRNAs to delete $100-150 \mathrm{bp}$ in the Runx 1 locus of mESCs and found that $23 \%$ of their clones had large deletions (up to $2 \mathrm{~kb}$ ) that escaped genotyping by short-range PCR (giving the impression that they were homozygous wildtype clones), with these complex on-target events becoming evident using long-range PCR (14). Similar damage and frequencies were also observed with the Cas9D10A nickase (14). More dramatic events were identified by Cullot et al., who CRISPR-targeted the UROS locus in HEK293T and K562 cells for HDR correction with a repair template (13). Their experiments suggest that CRISPR-Cas9 can induce mega-base scale chromosomal truncations ( $10 \%$ increase compared to controls). However, these cells have abnormal karyotypes and are p53 deficient, which may impact on their DNA damage repair machinery. In fact, they did not see the same effect in human foreskin fibroblasts but knocking-out of TP53 in these primary cells increased the large deletion events by 10 -fold (13). More recently, Przewrocka and colleagues observed a $6 \%$ incidence of chromosome arm truncations when targeting ZNF516 in p53-competent HCT116 cancer cell lines with CRISPR-Cas9, suggesting that TP53 expression alone may not predict predisposition of cells to large ontarget mutations (16).

Our laboratory used CRISPR-Cas9 genome editing to investigate the function of the pluripotency factor OCT4 (encoded by the POU5F1 gene on the p-arm of chromosome 6) during human preimplantation development (17). We generated a number of single-cell amplified genomic DNA (gDNA) samples for genotyping and confirmed on-target genome editing in all microinjected embryos and a stereotypic insertion/deletion (indel) pattern of mutations with the majority of samples exhibiting a $2 \mathrm{bp}$ deletion (17). However, we noted that in 5 of the samples analysed, the genotype could not be determined because of failures to PCR amplify the ontarget genomic fragment. This finding suggested complexity at the on-target region that may have abolished one or both PCR primer binding sites. Moreover, we identified that 57 of the 137 successfully genotyped samples (42\%) exhibited a homozygous wild-type genotype based on PCR amplification of a short genomic fragment (17). We originally interpreted these cases as unsuccessful targeting events, however, given the frequencies of the on-target complexities noted above, we speculated that our previous methods may have missed more complex on-target events.

Here, we have developed computational pipelines to analyse single-cell low-pass whole genome sequencing (WGS), transcriptome and deep-amplicon sequencing data to assess the prevalence of LOH events in the context of CRISPR-Cas9-edited early human embryos (Fig. S1). Our results indicate that LOH events on chromosome 6, including chromosomal and segmental copy number abnormalities, are more prevalent in OCT4-edited embryos compared to both Cas9-injected and uninjected controls, adding to the growing body of literature reporting that CRISPR-Cas9 genome editing can cause unintended on-target damage. Altogether, this underscores the importance of evaluating genome-edited samples for a diversity of mutations, including large-scale deletions, complex rearrangements and cytogenetic abnormalities, undetectable with methods that have routinely been used to interrogate targeted sites in previous studies. Our results sound a note of caution for the potential use of the CRISPR-Cas9 genome editing technology described here for reproductive purposes.

\section{Results \\ Segmental losses and gains at a CRISPR-Cas 9 on-target site identified by cytogenetics analysis}

In our previous study (17), in vitro fertilised zygotes donated as surplus to infertility treatment were microinjected with either an sgRNA-Cas9 ribonucleoprotein complex to target POU5F1 or Cas9 protein alone as a control and cultured for up to 6 days (targeted and control samples, respectively). We collected a single cell or a cluster of 2-5 cells from these embryos for cytogenetic, genotyping or transcriptomic analysis (Fig. S1).

To determine whether CRISPR-Cas9 genome editing leads to complex on-target DNA damage that would have been missed by our previous targeted amplicon sequencing, we reanalysed low-pass WGS data following whole-genome amplification (WGA) from 23 OCT4-targeted and 8 Cas9 control samples (SI Appendix, Table S1). Given the small sample size, we microinjected additional human embryos with a ribonucleoprotein complex to target POU5F1, or the Cas9 enzyme as a control, followed by single-cell WGA and low-pass WGS, as before (17). Here and below, the prefix that distinguishes the processing steps is followed by an embryo number and a cell number. The samples used for low-pass WGS were identified with prefix $L_{-}$(Fig. S1). The letter $C$ precedes the embryo number to distinguish CRISPR-Cas9 targeted from control samples (Fig. S1). Low-pass WGS data were used to generate copy number profiles for each sample to investigate the presence of abnormalities with a focus on chromosome 6 (Fig. 1A). As an additional comparison, we performed single- 
bioRxiv preprint doi: https://doi.org/10.1101/2020.06.05.135913; this version posted October 31, 2020. The copyright holder for this preprint (which was not certified by peer review) is the author/funder, who has granted bioRxiv a license to display the preprint in perpetuity. It is made available under aCC-BY-NC-ND 4.0 International license.

cell WGA and low-pass WGS of uninjected control embryos and distinguish these samples with a letter $U$ preceding the embryo number (Fig. S1)

After pre-processing and quality control, we examined the profiles of 65 samples (25 CRISPR-Cas 9 targeted, 16 Cas9 controls, and 24 uninjected controls Figs. S2A and S2B). 56 samples exhibited two copies of chromosome 6 with no obvious cytogenetic abnormalities (Figs. 1C, 1D and S3-S5). 17 of the CRISPR-Cas9 targeted samples, or $68 \%$, had no evidence of abnormalities on chromosome 6 . By contrast, we observed that 8 out of the 25 targeted samples had evidence of abnormalities on chromosome 6.4 targeted samples presented a segmental loss or gain that was directly adjacent to or within the POU5F1 locus on the p-arm of chromosome 6 (Figs. 1B, 1D and S5). Interestingly, this included two cells from the same embryo where one exhibited a segmental gain and the other a reciprocal loss extending from $6 \mathrm{p} 21.3$ to the end of $6 \mathrm{p}$ (Fig. 1B). Altogether, segmental abnormalities were detected in $16 \%$ of the total number of CRISPR-Cas9 targeted samples that were evaluated. We also observed that 4 targeted samples had evidence of a whole gain of chromosome 6 (Figs. 1B, 1D and S5), which also represents $16 \%$ of the targeted samples examined. Conversely, a single Cas9 control sample (6.25\%) had evidence of a segmental gain on the q-arm of chromosome 6, which was at a site distinct from the POU5F1 locus (Fig. S4). The uninjected controls did not display any chromosomal abnormalities (Figs. 1D and S3).

A

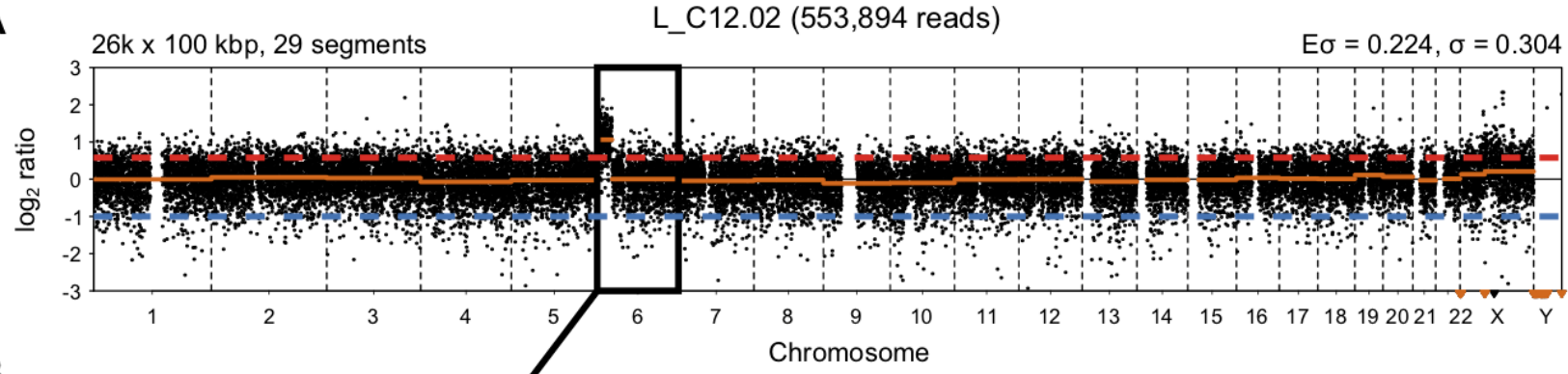

B

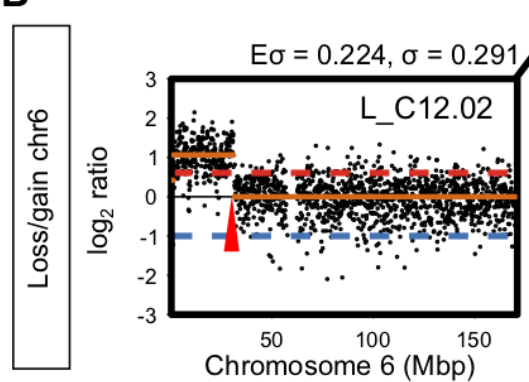

C

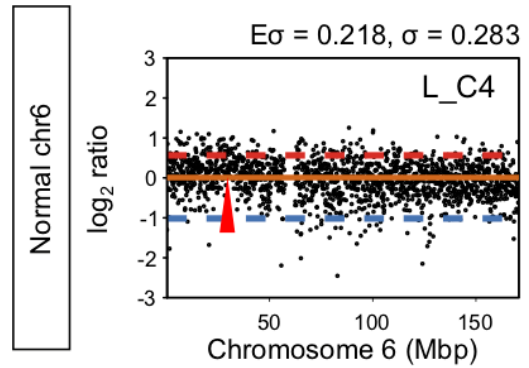

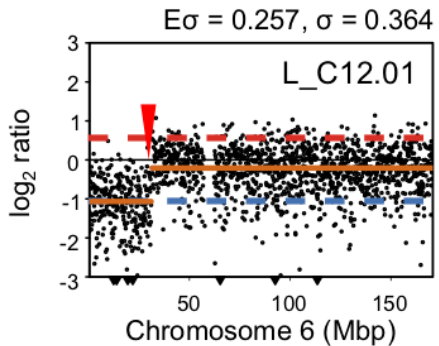

Chromosome $6(\mathrm{Mbp})$

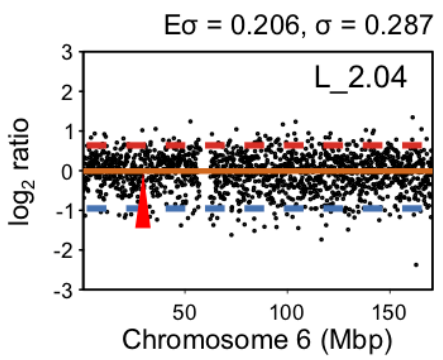

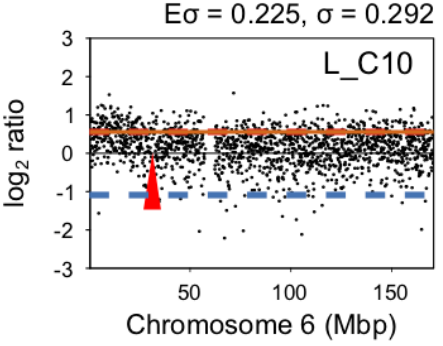

D

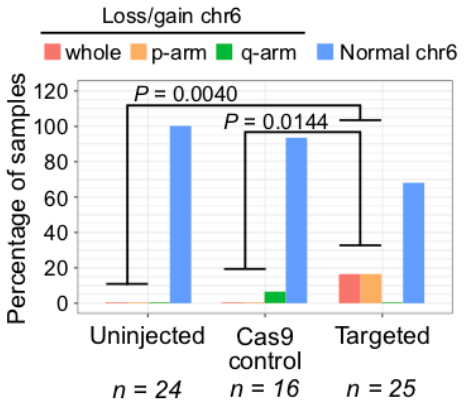

Fig. 1. Segmental losses/gains of chromosome 6 are prevalent in OCT4-targeted embryo samples. $(A)$ Copy number profile of sample L_C12.02. The segmental gain of chromosome 6 is highlighted. The profile was constructed with 26,000 bins of size $100 \mathrm{kbp}$, which produced 29 segments. The expected $(E \sigma)$ and measured $(\sigma)$ standard deviation of the profile are reported. $(B)$ Zoomed-in view of the copy number profile for samples with segmental losses or gains of chromosome 6. (C) Zoomed-in view of the copy number profile for samples with normal chromosome 6 . The Eo and $\sigma$ reported in $B$ and $\mathrm{C}$ correspond to the chromosome only. The approximate position of the POU5F1 gene is indicated by a red arrow. The red dashed line indicates a copy ratio of 3:2, while the blue dashed lines corresponds to a copy ratio of $1: 2$. (D) The percentage of control and targeted samples with whole or segmental losses/gains of chromosome 6 according to their copy number profiles. P-values are the result of two-tailed Fisher's tests.

The number of segmental and whole-chromosome abnormalities observed in the CRISPR-Cas9 targeted human cells was significantly different from that in the Cas9 $(P=0.0144$, two-tailed Fisher's test) and uninjected control ( $P=0.0040$, two-tailed Fisher's test) samples (Fig. 1D). Moreover, this significant difference 
can be attributed to the observed segmental abnormalities on $6 \mathrm{p}$, because excluding them from the comparison results in a negligible difference in whole-chromosome abnormalities between targeted and Cas 9 control samples $(P=0.1429$, two-tailed Fisher's test). This conclusion is further supported by the fact that none of the targeted samples show segmental losses or gains on the p-arm of chromosomes 5 and 7 , the closest in overall size to chromosome 6 , but the frequency of whole chromosome abnormalities is similar to that observed for chromosome 6, suggesting that genome editing does not exacerbate the rates of whole chromosome errors (Fig. S2C). The comparison we performed between Cas9 control and CRISPR-Cas9 genome edited samples includes a combination of both cleavage and blastocyst stage samples (Table S1). Because rates of aneuploidy are known to be significantly higher at the cleavage stage compared to the blastocyst (18), we wondered whether excluding the samples at the earlier cleavage stage would alter the conclusions drawn about the rates of aneuploidy in CRISPR-Cas9 targeted cells. Here, we found that in comparison to uninjected controls there remained a significantly higher proportion of chromosome 6 aneuploidies in OCT4-targeted cells collected at the blastocyst stage (Fig. S2D). Altogether, low-pass WGS analysis suggests that a significant proportion of unexpected on-target events leads to segmental abnormalities following CRISPR-Cas9 genome editing in human preimplantation embryos.

\section{Loss-of-heterozygosity identified by targeted deep sequencing}

The copy-number profiles described above with low-pass WGS data can only provide a coarse-grained karyotype analysis. To independently investigate the prevalence of $\mathrm{LOH}$ events at finer resolution and increased sequencing depth, we designed PCR primer pairs to amplify 15 fragments spanning a $20 \mathrm{~kb}$ region containing the POU5F1 locus. We also included a control PCR amplification in the ARGFX locus located on chromosome 3 (SI Appendix, Table S4). The PCR amplicons were used to perform deep sequencing by Illumina MiSeq using the gDNA isolated and amplified from 137 single cells or a cluster of 2-5 microdissected cells (111 CRISPR-Cas9 targeted and 26 Cas9 controls) (Fig. S1 and SI Appendix, Table S2). The prefix W_ distinguished samples whose gDNA was isolated solely for WGA and the prefix $G_{-}$was used to demarcate samples that underwent WGA via the G\&T-seq protocol (19). All of these samples were different from the samples used for the cytogenetic analyses above.

We then took advantage of the high coverage obtained at each of the sequenced fragments to call single nucleotide polymorphisms (SNPs), which allowed us to identify samples with putative LOH events: cases in which heterozygous variants, indicative of contribution from both parental alleles, cannot be confidently called in the amplicons flanking the CRISPR-Cas9 on-target site directly. Since we do not have the parental genotype from any of the samples that we analysed, we cannot exclude the possibility that they inherited a homozygous genotype. Therefore, we required the presence of heterozygous SNPs in at least one additional cell from the same embryo to call putative $\mathrm{LOH}$ events.

The variant-calling pipeline that we implemented was specifically adjusted for MiSeq data from single cell amplified DNA and includes stringent pre-processing and filtering of the MiSeq reads (Methods). To have sufficient depth of coverage and to construct reliable SNP profiles, we only considered samples with $\geq 5 x$ coverage in at least two thirds of the amplicons across the POU5F1 locus (Methods and Fig. S6A). This threshold allowed us to retain as many samples as possible and still be confident in SNP calling (20). In addition, we implemented a step in our SNP calling pipeline to control for allele overamplification bias, which is a common issue with single cell amplified DNA (21). This step changes homozygous calls to heterozygous if the fraction of reads supporting the reference allele is above the median value across samples (Figs. S6B and S6C and Methods). Thus, we proceeded with 42 CRISPR-Cas9 targeted and 10 Cas9 control samples with reliable SNP profiles for subsequent analysis. These data led to the identification of four different patterns: samples without clear evidence of $\mathrm{LOH}$, samples with $\mathrm{LOH}$ at the on-target site, bookended and open-ended LOH events (Fig. 2A and Figs. S7-S12).

In samples without LOH ( $20 \%$ of control and $11.9 \%$ of targeted samples), we were able to call heterozygous SNPs in multiple amplified fragments (G_8.04, G_C16.05 and W_C16.05, Fig. 2A). Cases with putative LOH at the locus have heterozygous SNPs in the amplicons covering exons 1 and 5 of the POU5F1 gene (fragments E1-2, G1 and E4 in Fig. 2A) and homozygous SNPs in between (50\% of control and $2.4 \%$ of targeted samples). These putative $\mathrm{LOH}$ samples would have had to have a cell isolated from the same embryo that had a detectable SNP(s) anywhere in between these flanking exons (e.g. see samples G_8.03 versus G_8.04 in Fig. S7). Interestingly, this was the most prevalent pattern in Cas9 control samples (Fig. 2B and Fig. S7), which may indicate the possibility of technical issues due to sequencing or overamplification of one parental allele (see below). Bookended samples have two heterozygous SNPs flanking the cut site but in fragments outside the POU5F1 locus ( $20 \%$ of control and $23.8 \%$ of targeted samples). These LOH events could represent 
deletions of lengths between $\sim 7 \mathrm{~kb}$ (G_C12.03, Fig. S10) and $\sim 12 \mathrm{~kb}$ (W_C11.04, Fig. S9). Finally, in openended samples ( $10 \%$ of control and $61.9 \%$ of targeted samples) it was not possible to find heterozygous SNPs in any of the amplified fragments (G_C12.07, Fig. 2A) or there was one or a few heterozygous SNPs on only one side of the region of interest (G_C16.02, Fig. S12). This was the most common pattern in targeted samples (Fig. 2B and Figs. S8-S12) and could represent large deletions of $\sim 20 \mathrm{~kb}$ in length (the size of the region explored) or larger.

A

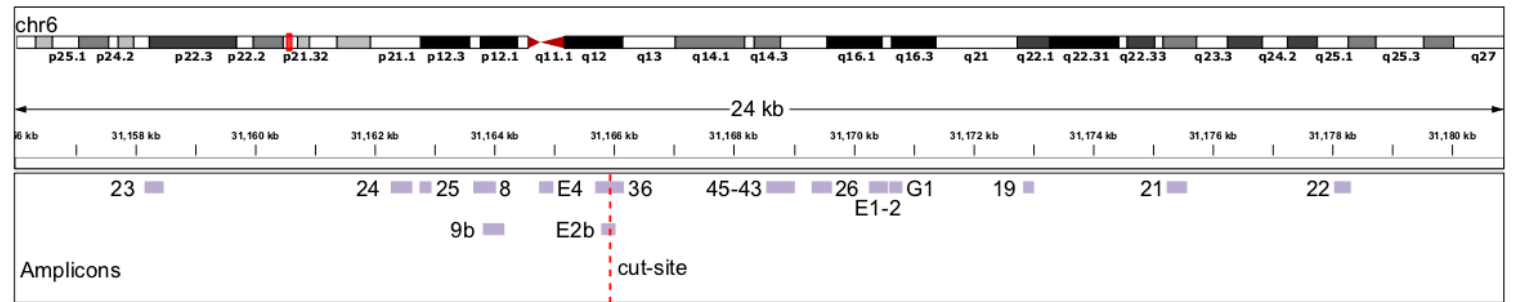

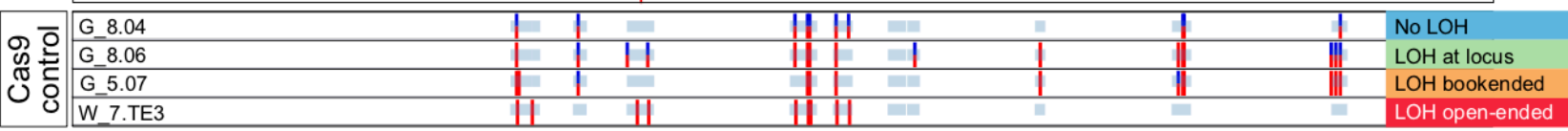

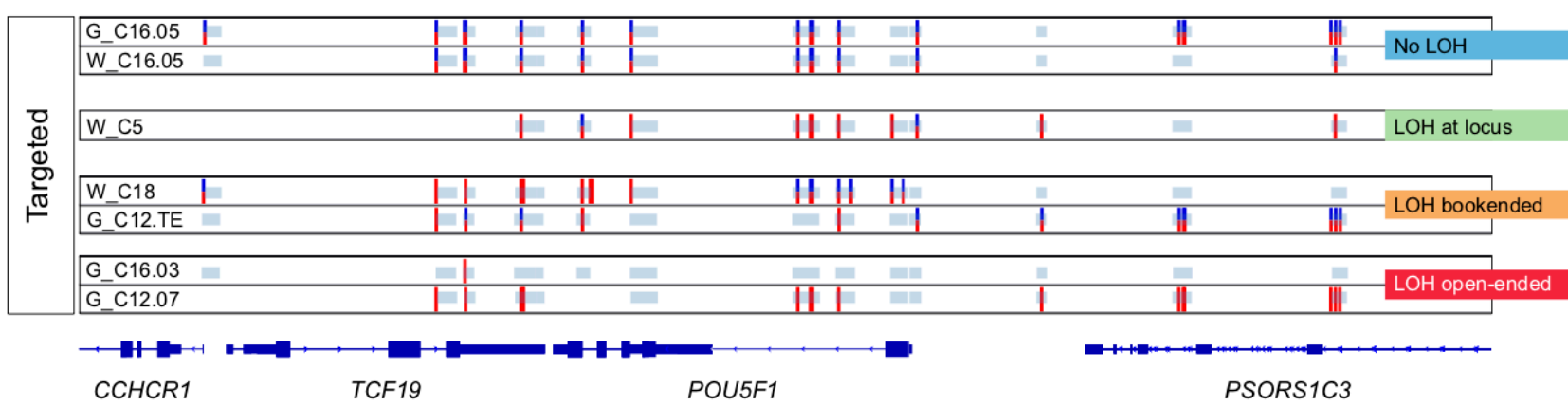

| Heterozygous SNP | Homozygous SNP $\quad$ Region with $\geq 5 x$ coverage

B

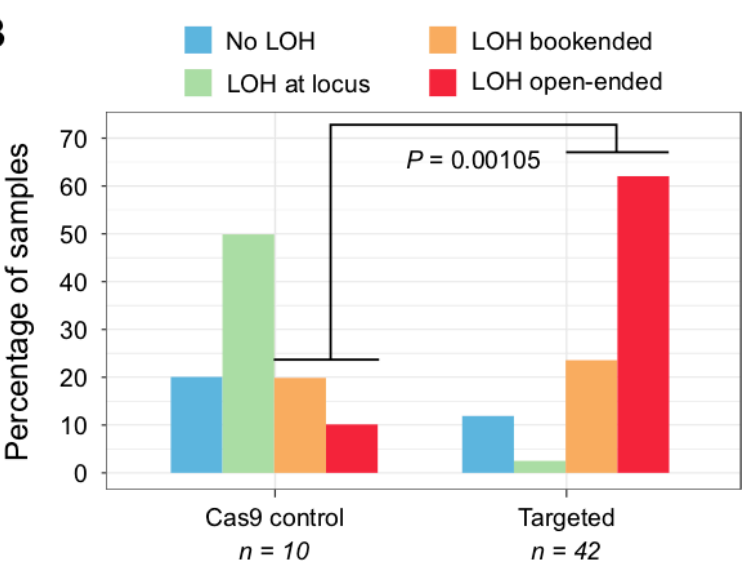

Fig. 2. LOH in the POU5F1 locus is prevalent among OCT4-targeted embryo samples. (A) Single nucleotide polymorphism (SNP) profiles constructed from deep sequencing of the depicted amplicons. The four types of loss-ofheterozygosity $(\mathrm{LOH})$ events observed are exemplified. Note that there are amplicons with $\geq 5 \mathrm{x}$ coverage in which SNPs were not called because all reads agree with the reference genome. (B) The frequency of each type of LOH event in control and targeted samples. P-value is the result of a two-tailed Fisher's test.

As mentioned above, the MiSeq data must be interpreted with caution given the presence of "LOH events" in Cas9 controls. The gDNA employed in these experiments was extracted and amplified with a kit based on multiple displacement amplification (MDA, Methods), which is common in single cell applications but is known to have high allelic dropout and preferential amplification rates (22). Even though, as mentioned above, we implemented a step to control for these biases, this estimate likely under-calls samples with heterozygosity. For example, some homozygous SNPs had 5\% of reads mapping to the reference allele but remained homozygous because they fall below the threshold that we used. Considering that we lack the parental genotypes as a reference to choose a more informed cut-off, our method to calculate one from the data 
represents an unbiased means to correct the presumed allele over-amplification in the samples. Moreover, we cannot exclude the possibility that the analysed single cells inherited a homozygous genotype in the explored region. Nevertheless, the fact that there is a significant number of CRISPR-Cas9 targeted samples with the

A

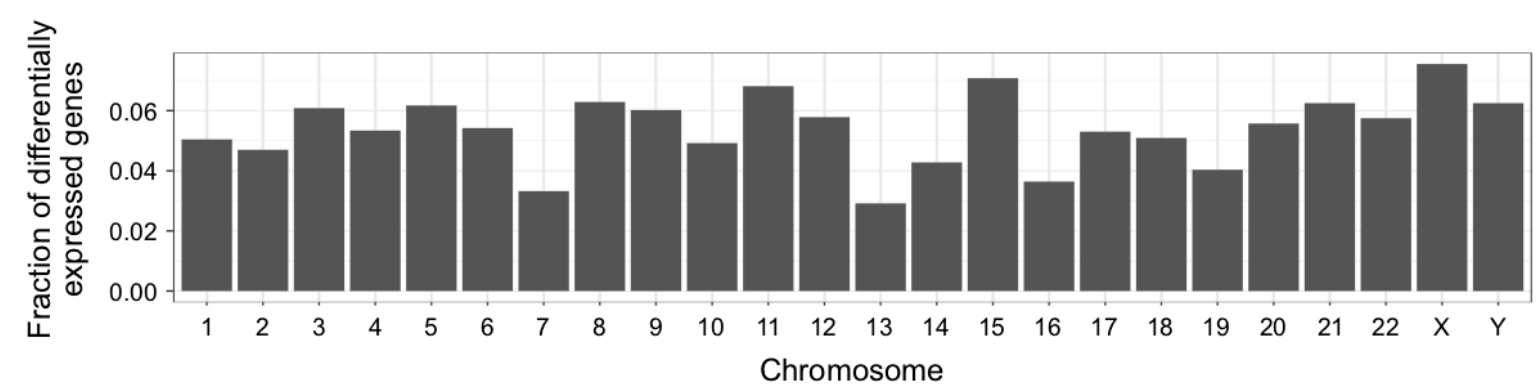

B

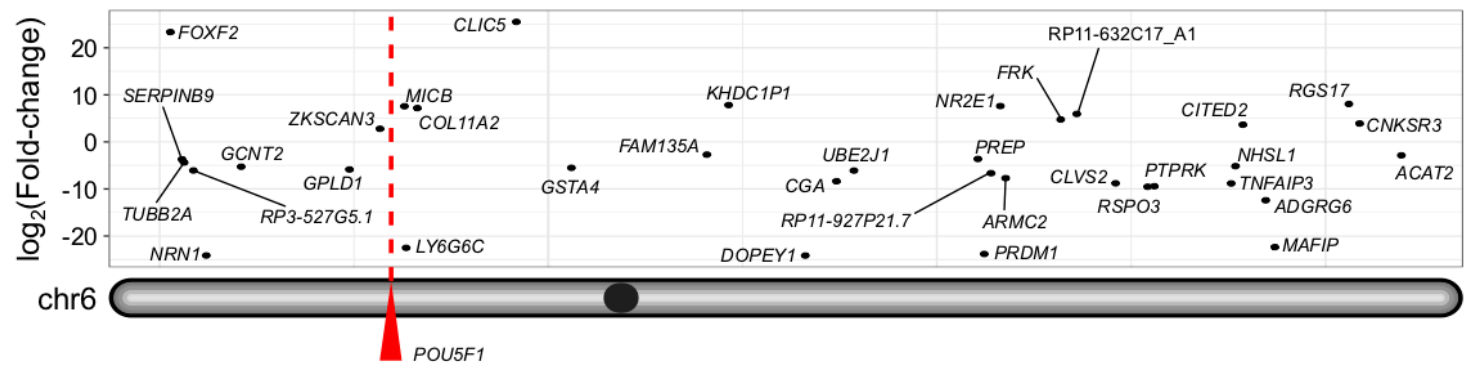

C

OCT4-null samples vs Cas9 controls

D

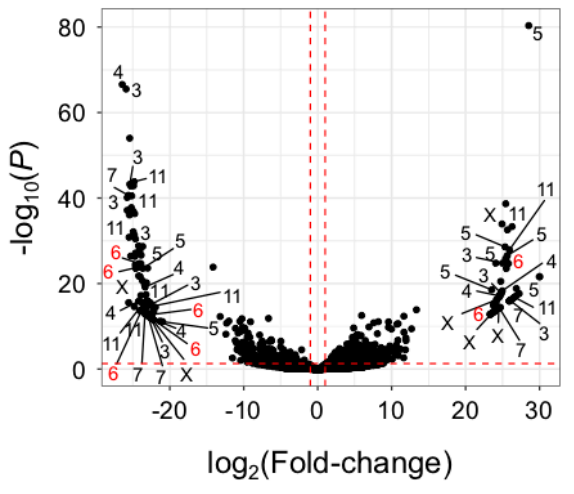
values are the result of two-tailed Fisher's tests.

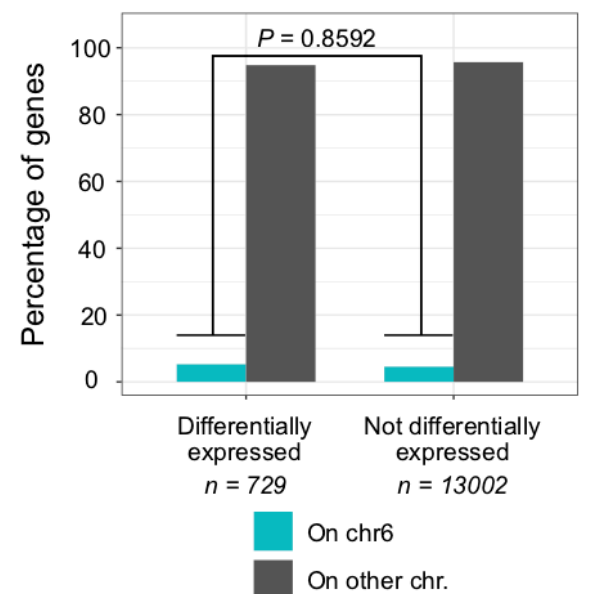

Fig. 3. LOH in OCT4-targeted samples does not lead to preferential misexpression of genes located on chromosome 6. (A) The fraction of differentially expressed genes per chromosome from the comparison between OCT4null samples and Cas9 controls. (B) Location of differentially expressed genes along chromosome 6 . (C) Volcano plot summarising the comparison between OCT4-null samples and Cas 9 controls with differential gene expression analysis. The chromosome location of some of the most dysregulated genes is shown (absolute log2 fold change $>20$ and Benjamini-Hochberg adjusted $P<0.05$ ). The red dashed lines correspond to absolute log2 fold changes $>1$ and BenjaminiHochberg adjusted $P<0.05$. (D) Genes located on chromosome 6 are not overrepresented in the list of loci whose expression is disturbed upon OCT4 knock out. The same applies for genes directly upstream to the POU5F1 gene. P-

\section{Unexpected CRISPR-Cas9-induced on-target events do not lead to preferential misexpression of telomeric to POU5F1 genes}

Our low-pass WGS and SNP analysis above indicate mutations at the POU5F1 locus that are larger than discrete indels. We therefore wondered if this on-target complexity may encompass the mutations of genes adjacent or telomeric to POU5F1 that could complicate the use of CRISPR-Cas9 to understand gene function in human development or other contexts where the analysis of primary cells is required. To address this, we reanalysed the single-cell RNA sequencing (scRNA-seq) transcriptome datasets (Table S6) we generated 
previously (17) and focused on the chromosome location of transcripts (Figs. 3A-C). This analysis indicated that differentially expressed genes are not biased to a specific chromosome (Fig. 3A). Moreover, differentially expressed genes are not enriched to either chromosome 6 or the region telomeric to the CRISPR-Cas9 ontarget site (Fig. 3D). These results suggest that the transcriptional differences observed as a consequence of POU5F1 targeting are not confounded by mutations of genes adjacent, or telomeric, to the on-target locus. This could be due to a number of reasons. For example, given that the proportion of samples that exhibit unintended CRISPR-Cas9-induced mutations (e.g. segmental aneuploidies or LOH events) is low, the sample size used is sufficiently high to mask any transcriptional differences in genes adjacent to the cut site in samples with segmental loss of the $\mathrm{p}$-arm of chromosome 6 . It is also possible that the extent of the on-target complexity is exaggerated using the gDNA-based pipelines we developed. Notably, because we use single-cell samples, as mentioned above, these are prone to allele over-amplification and this can confound the interpretation of on-target mutation complexity.

\section{No evidence of on-target complexity using digital karyotype and LOH analysis of the single-cell transcriptome data}

The use of RNA-seq data to detect chromosomal abnormalities (23) has great potential to complement the informative low-pass WGS or array CGH methods currently used for embryo screening in the context of assisted reproductive technologies $(24,25)$. In addition to karyotype analysis, transcriptome data may also provide information about embryo competence at the molecular level. Groff and colleagues have demonstrated that aneuploidy can be estimated based on significant variations in gene expression in the affected chromosome(s) compared to reference control samples (24). In addition, Weissbein et al. developed a pipeline, called eSNP-Karyotyping, for the detection of LOH in chromosome arms (26). eSNP-Karyotyping is based on measuring the ratio of expressed heterozygous to homozygous SNPs. We applied these two approaches, hereinafter referred to as z-score- and eSNP-Karyotyping, to the single-cell RNA-seq (scRNA-seq) samples (distinguished with the prefix T_) obtained using the G\&T-seq protocol (14) (SI Appendix, Table S3). This allowed us to investigate whether transcriptome data could be used to determine the frequency of LOH events in CRISPR-Cas9 targeted embryos.

A

\begin{tabular}{|c|c|}
\hline Control Targeted \\
\hline
\end{tabular}

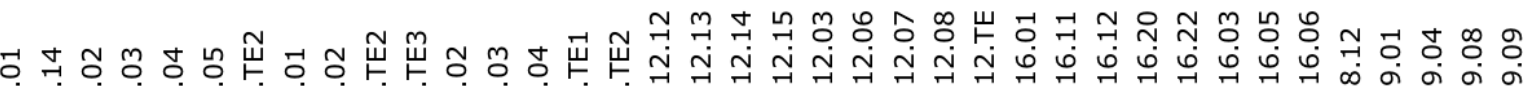

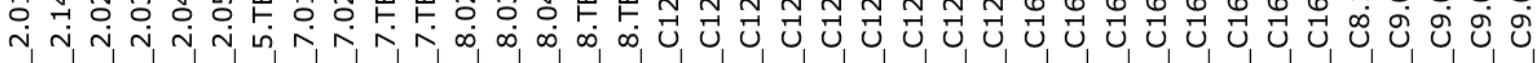

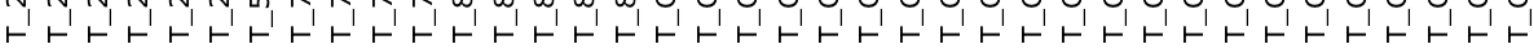
$\operatorname{chr6}\left[\begin{array}{c}p \\ q\end{array}\right]$

r 1 -

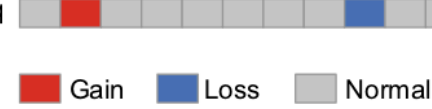

B

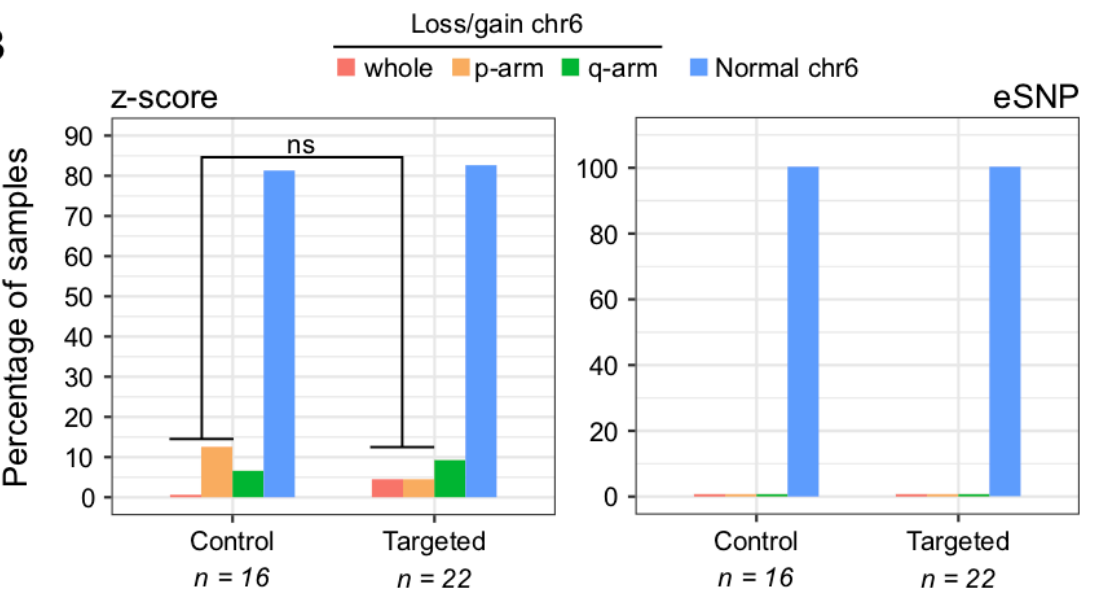

Fig. 4. Transcriptome-based karyotypes do not capture segmental losses/gains of chromosome 6 in OCT4targeted embryo samples. $(A)$ Digital karyotype based on the total gene expression deviation from the average of each chromosome arm (z-score-karyotyping). Only chromosome 6 (see Fig. S14A for the rest of the chromosomes). (B) The percentage of control and targeted samples with segmental losses/gains of chromosome 6 according to their transcriptome-based karyotype (see Figs. S14A and S15). P-value is the result of a two-tailed Fisher's test. 
Since eSNP-Karyotyping relies on SNP calls from gene expression data, it is very sensitive to depth and breadth of sequencing (26). Therefore, we used results from this method as a reference to select high quality samples for our transcriptome-based analyses (Fig. S13A-C). After these filtering steps, we retained 38 samples (22 CRISPR-Cas9 targeted and 16 Cas9 controls) to analyse further.

In general, we found good agreement between the chromosomal losses detected by z-score-karyotyping and the LOH events identified by eSNP-Karyotyping (Fig. S14A and S14B). For example, the digital karyotype of Fig. S14A shows the loss of chromosome 4, the p-arm of chromosome 7 and the q-arm of chromosome 14 in sample T_7.01, as well as the loss of chromosome 3 and the p-arm of chromosome 16 in sample T_C16.06. These abnormalities are identified as LOH events in the eSNP-Karyotyping profiles of the same samples (Fig. 14B). Moreover, the copy number profiles built from low-pass WGS data for different cells from the same embryos also corroborates these chromosomal abnormalities (Fig. S13D and S13E). In terms of events that could be associated with CRISPR-Cas9 on-target damage, z-score-karyotyping identified the loss of chromosome 6 in sample T_C12.07 (Fig. 4A), which is consistent with the open-ended LOH pattern observed in the gDNA extracted from the same cell G_C12.07 (Fig. S10) and the segmental loss detected in sample L_C12.01 from the same embryo (Fig. 1B). Also, the gain of the p-arm of chromosome 6 was detected in sample T_C12.15 (Fig. 4A), which is consistent with the segmental gain observed in sample L_C12.02 from the same embryo (Fig. 1B). The gains and losses of chromosome 6 in samples T_2.02, T_2.03, T_2.14, T_7.02 and T_C16.06 (Fig. 4A) are difficult to interpret due to the low quality of their MiSeq data or the lack of amplicon information for the q-arm (Fig. S7 and Fig. S12). Interestingly, eSNP-Karyotyping did not detect any LOH events in chromosome 6 (Fig. S15), suggesting that this approach is not sensitive enough to detect segmental abnormalities in single cell samples. Overall, the transcriptome-based karyotypes did not confirm the trends observed in the gDNA-derived data (Fig. 4B).

\section{Discussion}

In all, we reveal unexpected on-target complexity following CRISPR-Cas9 genome editing of human embryos. Our data suggest approximately $16 \%$ of samples exhibit segmental losses/gains adjacent to the POU5F1 locus and $\mathrm{LOH}$ events that span $4 \mathrm{~kb}$ to at least $20 \mathrm{~kb}$. Chromosome instability, including whole or segmental chromosome gain or loss, is common in human preimplantation embryos $(27,28)$. However, in contrast to Cas9 control embryos, we noted a significantly higher frequency of CRISPR-Cas9 targeted embryos with a segmental gain or loss that was directly adjacent to the POU5F1 on-target site. The segmental errors were observed in embryos from distinct genetic backgrounds and donors. Therefore, together with their on-target location, this suggests that the errors may have been an unintended consequence of CRISPR-Cas 9 genome editing. This is supported by the higher frequency of larger LOH events that we observed in CRISPR-Cas9 targeted embryos compared to Cas 9 controls using an independent targeted deep-sequencing approach. However, due to the nature of our datasets (shallow sequencing, MDA-amplified gDNA, lack of parental genotypes) we may be overestimating LOH events. This may explain some of the on-target complexity observed in Cas9 control samples but does not account for the significantly higher proportion of LOH in the CRISPR-Cas 9 targeted samples. It is important to note that $68 \%$ of CRISPR-Cas 9 targeted cells did not exhibit any obvious segmental or whole chromosome 6 abnormalities, indicating that their genotype and phenotype, with respect to OCT4 function, are interpretable. Moreover, our transcriptome-based digital karyotypes and differential gene expression analysis indicate biallelic transcripts and gene expression up- and down-stream of the POU5F1 locus in so far as is resolvable from scRNA-seq data, suggesting that in these samples the $\mathrm{LOH}$ does not lead to the misexpression of other genes adjacent to the POU5F1 locus. Also, our work and previous accounts of unexpected CRISPR-Cas9 editing outcomes $(9,10,12-14,16)$ indicate that the frequency of discrete on-target events predominates, which should increase the confidence of the interpretation of functional studies in human embryos. Given the likelihood of mosaicism, it is unclear whether the segmental abnormalities we observed in any one cell analysed from each embryo are representative of the entire CRISPR-Cas9 targeted embryo or a subset of cells within the embryo. Altogether, this points to the need to use robust techniques to distinguish cells affected by on-target complexity and large deletions following CRISPR-Cas9-mediated genome editing from cells with less complex mutations and our computational pipelines and multi-omics analyses are approaches that may be used in the future.

By contrast, we did not observe significantly more abnormalities on chromosome 6 using methods to determine $\mathrm{LOH}$ or karyotype from scRNA-seq datasets. There are several factors that could account for the discrepancy between these datasets. Firstly, we do not have the transcriptome from the same samples that showed gains and losses of chromosome 6 in the cytogenetics analysis. A follow-up study in which both transcriptomics and 
cytogenetics data are extracted from the same sample would be very informative and could be performed by modifying the G\&T-seq protocol (19) to incorporate a multiple annealing and looping-based amplification cycles (MALBAC) method for WGA (29) in place of MDA, which was used here due to the proofreading activity of the phi29 MDA polymerase at the expense of high preferential amplification rates (22). Secondly, mosaicism is common in human preimplantation embryos (30) and this could explain why the digital karyotypes based on gene expression did not detect abnormalities at the same rate as the copy number profiles. Another possibility is that the LOH events are not sufficiently large to impact total gene expression of chromosome 6, which is what z-score- and eSNP-Karyotyping rely on. This could also account for the cytogenetics results, as LOH up to a few $\mathrm{Mb}$ in size could cause mapping issues due to the very low coverage of shallow sequencing that are reflected as gains and losses of whole chromosome segments. Finally, the LOH events detected by gDNAderived data may only affect genes that are not expressed in the embryo context or whose expression is so low that it cannot be accurately measured by scRNA-seq. So, when z-score- and eSNP-Karyotyping compare gene or SNP expression of targeted versus control samples, no significant differences are identified.

The segmental aneuploidies identified by cytogenetics analysis (Figs. 1B and S3-S5) most probably point to the occurrence of complex genomic rearrangements in OCT4-targeted samples, such as chromosomal translocations or end-to-end fusions, as it seems unlikely that the rest of the chromosome would continue to be retained without a telomere (31-33). It is likely that human embryos tolerate aneuploidy up to embryo genome activation, given that even embryos with observed multipolar spindles continue to develop during early cleavage divisions (34). Following this, chromosomal anomalies are likely to become increasingly detrimental to cellular viability, although a degree of tolerance may persist in trophectoderm cells (28). Why early embryos fail to arrest despite chaotic chromosomal errors such as multipolar spindle formation or presumptive unresolved double strand breaks following CRISPR-Cas9 genome editing is unclear and crucial to understand. An important next step to gain insights into the extent of the damage would be to use alternative methods. One possibility to understand the complexity would be to perform cytogenetic analysis using fluorescence in situ hybridization (FISH) (35) to probe for segments of chromosome 6. Another option is a chromosome walk-along approach to amplify genomic fragments even further away from the $20 \mathrm{~kb}$ genomic region that we evaluated, in order to bookend heterozygous SNPs on either side of the POU5F1 on-target site. This may be kilo- or mega-bases away from the on-target site based on previous publications in the mouse or human cell lines $(9$, $10,12-14)$.

Based on our data, the possibility of gene editing via IH-HR cannot be definitely excluded. A pre-print by Liang et al. (36) suggests that IH-HR could be one of the major DNA double-strand break repair pathways in human embryos. Following a similar approach to their previous study (8), the authors used CRISPR-Cas9-mediated genome editing to target a paternal mutation and were able to amplify an $\sim 8 \mathrm{~kb}$ genomic DNA fragment which, together with G-banding and FISH of ESCs derived from targeted embryos, suggests that repair from the maternal chromosome by IH-HR results in a stretch of $\mathrm{LOH}$. Of note, due to the selection bias that occurs during ESC derivation and the mosaicism observed following genome editing, it is not possible to draw definitive conclusions about the extent of $\mathrm{LOH}$ or its cause in an embryo context, whereby cells with complex mutations may be preferentially excluded from ESC derivation. By contrast, another pre-print by Zuccaro et al. using the same microinjection method suggests that the LOH observed following CRISPR-Cas9-mediated genome editing is a consequence of whole chromosome or segmental loss adjacent to the on-target site and that microhomology-mediated end-joining (MMEJ) is the dominant repair pathway in this context (37). This corroborates our previous findings in human embryos targeted post-fertilisation, where we noted a stereotypic pattern to the type of indel mutations and speculated that this was likely due MMEJ (17). Although microhomologies can promote gene conversion by, for example, inter-chromosomal template switching in a RAD51-dependent manner (38), based on our previous transcriptome analysis, we found that components of the MMEJ pathway (i.e. $P O L Q$ ) are transcribed in early human embryos, while factors essential for HDR (i.e. $R A D 51)$ are not appreciably expressed. This suggests that MMEJ-derived large deletions $(14,37)$ are more likely than microhomology-mediated gene conversion in this context, though protein expression has yet to be fully characterised. Consistent with this, a significant fraction of somatic structural variants arises from MMEJ in human cancer (39). Moreover, microhomology-mediated break-induced replication underlies copy number variation in mammalian cells (40) and microhomology/microsatellite-induced replication leads to segmental anomalies in budding yeast (41). The discrepancy between the Liang et al. and Zuccaro et al. studies could be due to locus-dependent differences of CRISPR-Cas9 genome editing fidelity. For example, Przewrocka et al. demonstrate that the proximity of the CRISPR-Cas9-targeted locus to the telomere significantly increases the possibility of inadvertent chromosome arm truncation (16). To fully elucidate the $\mathrm{LOH}$ that has occurred at the on-target site in our study, and to resolve the controversy over the IH-HR reported by others $(8,9,36,37)$, will require the development of a pipeline to enrich for the region of interest and then perform deep (long-read) sequencing to evaluate the presence and extent of on-target damage. By bookending SNPs on either side of 
an LOH event, primers could be designed to incorporate the SNPs and ensure that both parental alleles are amplified. However, this is difficult to perform, and alternative methods include using CRISPR gRNAs to cut just outside of the $\mathrm{LOH}$ region followed by long-read sequencing (42).

It would also be of interest to evaluate whether other genome editing strategies, such as prime and base editing, nickases or improvements in the efficiency of integrating a repair template, may reduce the on-target complexities observed by us and others using spCas9. However, non-negligible frequencies of editingassociated large deletions have been reported after the use of the Cas9D10A nickase in MESCs (14) and prime editing in early mouse embryos (43). By contrast, while proof-of-principle studies suggest that base editors could be used to repair disease-associated mutations in human embryos, further refinements to reduce the likelihood of unexpected conversion patterns and high rates of off-target edits would be of benefit (2). There are too few studies to date using repair templates. Of the studies that have been conducted, the reported efficiencies of repair with templates in human embryos are very low $(5,7,8)$. Modulation of DNA damage repair factors or tethering Cas 9 enzymes with a repair template may yield improvements that could allow for the control of editing outcomes.

Our re-evaluation of on-target mutations, together with previous accounts of unexpected CRISPR-Cas9 ontarget damage $(9,10,12-14)$, strongly underscores the importance of further basic research in a number of cellular contexts to resolve the damage that occurs following genome editing. Moreover, this stresses the significance of ensuring whether one or both parental chromosome copies are represented when determining the genotype of any sample to understand the complexity of on-target CRISPR mutations, especially in human primary cells.

\section{Methods}

Ethics statement. We reprocessed the DNA and reanalysed the data generated in our previous study (17). This corresponds to 168 samples (134 OCT4-targeted and 34 Cas 9 controls) across 32 early human embryos (24 OCT4-targeted and 8 Cas9 controls). For the present work, we used 56 additional single-cell samples (19 OCT4-targeted, 12 Cas9 controls and 25 uninjected controls) across 22 early human embryos (1 OCT4targeted, 1 Cas 9 control and 20 uninjected controls). This study was approved by the UK Human Fertilisation and Embryology Authority (HFEA): research licence number R0162, and the Health Research Authority's Research Ethics Committee (Cambridge Central reference number 19/EE/0297). Our research is compliant with the HFEA code of practice and has undergone inspections by the HFEA since the licence was granted. Before giving consent, donors were provided with all of the necessary information about the research project, an opportunity to receive counselling and the conditions that apply within the licence and the HFEA Code of Practice. Specifically, patients signed a consent form authorising the use of their embryos for research including genetic tests and for the results of these studies to be published in scientific journals. No financial inducements were offered for donation. Patient information sheets and the consent documents provided to patients are publicly available (https://www.crick.ac.uk/research/a-z-researchers/researchers-k-o/kathyniakan/hfea-licence/). Embryos surplus to the IVF treatment of the patient were donated cryopreserved and were transferred to the Francis Crick Institute where they were thawed and used in the research project.

CRISPR-Cas9 targeting of POU5F1. We analysed single cells or trophectoderm biopsies from human preimplantation embryos that were CRISPR-Cas9 edited in our previous study (17) plus an additional 56 samples used in the present work. In vitro fertilised zygotes donated as surplus to infertility treatment were microinjected with either a sgRNA-Cas9 ribonucleoprotein complex or with Cas9 protein alone and cultured for 5-6 days (targeted and control samples, respectively). The sgRNA was designed to target exon 2 of the POU5F1 gene and experiments performed as previously described (17). Genomic DNA from Cas9 control and OCT4-targeted was isolated using the REPLI-g Single Cell Kit (QIAGEN, 150343). DNA samples isolated for cytogenetic analysis were amplified with the SurePlex Kit (Rubicon Genomics). See the SI Appendix for more details.

Cytogenetic analysis. Low-pass whole genome sequencing (depth of sequencing $<0.1 \mathrm{x}$ ) libraries were prepared using the VeriSeq PGS Kit (Illumina) or the NEB Ultra II FS Kit and sequenced with the MiSeq platform as previously described (17) or with Illumina HiSeq 4000, respectively. Reads were aligned to the human genome hg19 using BWA v0.7.17 (44) and the copy number profiles generated with QDNAseq v1.24.0 (45). See the SI Appendix for more details.

PCR primer design and testing. PCR primer pairs were designed with the Primer3 webtool 
primer temperature settings: $\mathrm{Min}=56, \mathrm{Opt}=58$, Max $=60$. We tested all primers using $1 \mathrm{uL}$ of genomic DNA from H9 human ES cells in a PCR reaction containing 12.5 uL Phusion High Fidelity PCR Master Mix (NEB, M0531L), $1.25 \mathrm{uL} 5 \mathrm{uM}$ forward primer, $1.25 \mathrm{uL} 5 \mathrm{uM}$ reverse primer and 9 uL nuclease-free water. Thermocycling settings were: $95^{\circ} \mathrm{C} 5 \mathrm{~min}, 35$ cycles of $95^{\circ} \mathrm{C} 30 \mathrm{~s}, 58^{\circ} \mathrm{C} 30 \mathrm{~s}, 72^{\circ} \mathrm{C} 1 \mathrm{~min}$, and a final extension of $72^{\circ} \mathrm{C} 5 \mathrm{~min}$. We confirmed the size of the PCR products by gel electrophoresis. See the SI Appendix for more details.

PCR amplification and targeted deep sequencing. Isolated DNA was diluted 1:100 in nuclease-free water. We used the QIAgility robot (QIAGEN, 9001531) for master mix preparation (see above) and distribution to 96-well plates (Table S5). Then, the Biomek FX liquid handling robot (Beckman Coulter, 717013) was used to transfer 1uL of DNA to the master mix plates and to mix the reagents. The PCR reaction was run with the settings described above. PCR products were cleaned with the Biomek FX robot using the chemagic SEQ Pure20 Kit (PerkinElmer, CMG-458). Clean PCR amplicons from the same DNA sample were pooled to generate 137 libraries that were sequenced by Illumina MiSeq v3. See the SI Appendix for more details.

SNP-typing. We trimmed the MiSeq paired-end reads with DADA2 (46), corrected substitution errors in the trimmed reads with RACER (47) and mapped the corrected reads to the human genome hg38 with BWA v0.7.17 (44). Subsequently, SAM files were converted to the BAM format and post-processed using Samtools v1.3.1 (48). SNP calling was performed with BCFtools v1.8 (49) using mpileup and call. SNPs supported by less than 10 reads and with mapping quality below 50 were filtered out. To control for allele overamplification, homozygous SNPs were changed to heterozygous if the fraction of reads supporting the reference allele was at least $6 \%$ of the total (21). This threshold corresponds to the median of the distribution of the fraction of reads supporting the reference allele across samples. See the SI Appendix for more details.

scRNA-seq data analysis. scRNA-seq reads from G\&T-seq samples were processed as previously described (17). Samples with a breadth of sequencing below 0.05 were not considered for any downstream analysis (Fig. S13A-C). Differential gene expression analysis was carried out with DESeq2 v1.10.1 (50). For digital karyotyping based on gene expression, we adapted the method described in (24) to identify gains or losses of chromosomal arms (z-score-karyotyping). For digital karyotyping based on SNP expression, we applied the eSNP-Karyotyping pipeline with default parameters (26). See the SI Appendix for more details.

Data and software availability. All data supporting the findings of this study are available within the article and its supplementary information. MiSeq and low-pass WGS data have been deposited to the Sequence Read Archive (SRA) under accession number PRJNA637030. scRNA-seq data was extracted from the Gene Expression Omnibus (GE) using accession GSE100118. A detailed analysis pipeline is available at the following site: https://github.com/galanis//loh scripts.

\section{Acknowledgements}

We thank the generous donors whose contributions have enabled this research. We thank Robin Lovell-Badge, James Haber, Alexander Frankell, Aska Przewrocka, Charles Swanton, Maxime Tarabichi, the Niakan and Turner laboratories for discussion, advice and feedback; the Francis Crick Institute's core facilities including Jerome Nicod and Robert Goldstone at the Advanced Sequencing Facility; D.W. was supported by the National Institute for Health Research (NIHR) Oxford Biomedical Research Centre Programme. N.K. was supported by the University of Oxford Clarendon Fund and Brasenose College Joint Scholarship. Work in the K.K.N. and J.M.A.T. labs was supported by the Francis Crick Institute, which receives its core funding from Cancer Research UK (FC001120 and FC001193), the UK Medical Research Council (FC001120 and FC001193), and the Wellcome Trust (FC001120 and FC001193). Work in the K.K.N. laboratory was also supported by the Rosa Beddington Fund.

\section{Author contributions}

K.K.N. conceived the project. N.M.E.F. generated the genomics and transcriptomics datasets. A.M., E.H. and G.A-L. designed and tested primers. N.K. and D.W. generated the low-pass WGS data. M.G. performed the PCR amplification experiments with the robotics equipment. J.Z. implemented the variant calling pipeline for the amplicon sequencing data. G.A-L. collected, processed and analysed all the datasets. J.M.A.T. provided advice on the project design. G.A-L. and K.K.N. wrote the manuscript with the help from all other authors. All authors assisted with experimental design and figures. 


\section{References}

1. M. Adli, The CRISPR tool kit for genome editing and beyond. Nature Communications 9, 1911 (2018).

R. A. Lea, K. K. Niakan, Human germline genome editing. Nature Cell Biology 21, 1479-1489 (2019).

3. National Academy of Medicine, National Academy of Sciences, The Royal Society, Heritable Human Genome Editing (The National Academies Press, 2020) https:/doi.org/10.17226/25665.

4. Nuffield Council on Bioethics, Genome editing and human reproduction: social and ethical issues (Nuffield Council on Bioethics, 2018).

5. P. Liang, et al., CRISPR/Cas9-mediated gene editing in human tripronuclear zygotes. Protein \& Cell 6, 363-372 (2015).

6. X. Kang, et al., Introducing precise genetic modifications into human 3PN embryos by CRISPR/Casmediated genome editing. Journal of Assisted Reproduction and Genetics 33, 581-588 (2016).

7. L. Tang, et al., CRISPR/Cas9-mediated gene editing in human zygotes using Cas9 protein. Molecular Genetics and Genomics 292, 525-533 (2017).

8. H. Ma, et al., Correction of a pathogenic gene mutation in human embryos. Nature 548, 413-419 (2017).

9. D. Egli, et al., Inter-homologue repair in fertilized human eggs? Nature 560, E5-E7 (2018).

10. F. Adikusama, et al., Large deletions induced by Cas9 cleavage. Nature 560, E8-E9 (2018).

11. H. Ma, et al., Ma et al. reply. Nature 560, E10-E16 (2018).

12. M. Kosicki, K. Tomberg, A. Bradley, Repair of double-strand breaks induced by CRISPR-Cas9 leads to large deletions and complex rearrangements. Nature Biotechnology 36, 765-771 (2018).

13. G. Cullot, et al., CRISPR-Cas9 genome editing induces megabase-scale chromosomal truncations. Nature Communications 10 (2019).

14. D. D. G. Owens, et al., Microhomologies are prevalent at Cas9-induced larger deletions. Nucleic Acids Research 47, 7402-7417 (2019).

15. H. Lee, J.-S. Kim, Unexpected CRISPR on-target effects. Nature Biotechnology 36, 703-704 (2018).

16. J. Przewrocka, A. Rowan, R. Rosenthal, N. Kanu, C. Swanton, Unintended on-target chromosomal instability following CRISPR/Cas9 single gene targeting. Annals of Oncology 31, 1270-1273 (2020).

17. N. M. E. Fogarty, et al., Genome editing reveals a role for OCT4 in human embryogenesis. Nature 550, 67-73 (2017).

18. E. Fragouli, S. Alfarawati, K. Spath, D. Wells, Morphological and cytogenetic assessment of cleavage and blastocyst stage embryos. Molecular Human Reproduction 20, 117-126 (2014).

19. I. C. Macaulay, et al., G\&T-seq: parallel sequencing of single-cell genomes and transcriptomes. Nature Methods 12, 519-522 (2015).

20. T. Kishikawa, et al., Empirical evaluation of variant calling accuracy using ultra-deep whole-genome sequencing data. Scientific Reports 9, 1784 (2019).

21. N. Kubikova, et al., Clinical application of a protocol based on universal next-generation sequencing for the diagnosis of beta-thalassaemia and sickle cell anaemia in preimplantation embryos. Reproductive BioMedicine Online 37, 136-144 (2018).

22. E. Borgström, M. Paterlini, J. E. Mold, J. Frisen, J. Lundeberg, Comparison of whole genome amplification techniques for human single cell exome sequencing. PLOS ONE 12, e0171566 (2017).

23. J. A. Griffiths, A. Scialdone, J. C. Marioni, Mosaic autosomal aneuploidies are detectable from singlecell RNAseq data. BMC Genomics 18, 904 (2017).

24. A. F. Groff, et al., RNA-seq as a tool for evaluating human embryo competence. Genome Res. 29, 1705-1718 (2019).

25. M. Poli, et al., Past, Present, and Future Strategies for Enhanced Assessment of Embryo's Genome and Reproductive Competence in Women of Advanced Reproductive Age. Frontiers in Endocrinology 10, 154 (2019).

26. U. Weissbein, M. Schachter, D. Egli, N. Benvenisty, Analysis of chromosomal aberrations and recombination by allelic bias in RNA-Seq. Nature Communications 7, 12144 (2016).

27. E. Vanneste, et al., Chromosome instability is common in human cleavage-stage embryos. Nature Medicine 15, 577-583 (2009).

28. D. Babariya, E. Fragouli, S. Alfarawati, K. Spath, D. Wells, The incidence and origin of segmental aneuploidy in human oocytes and preimplantation embryos. Human Reproduction 32, 2549-2560 (2017).

29. C. Zong, S. Lu, A. R. Chapman, X. S. Xie, Genome-Wide Detection of Single-Nucleotide and CopyNumber Variations of a Single Human Cell. Science 338, 1622-1626 (2012).

30. R. C. McCoy, Mosaicism in preimplantation human embryos: when chromosomal abnormalities are the norm. Trends in Genetics 33, 448-463 (2017).

31. B. van Steensel, A. Smogorzewska, T. de Lange, TRF2 Protects Human Telomeres from End-to-End Fusions. Cell 92, 401-413 (1998). 
32. A. W. I. Lo, et al., Chromosome Instability as a Result of Double-Strand Breaks near Telomeres in Mouse Embryonic Stem Cells. Molecular and Cellular Biology 22, 4836-4850 (2002).

33. R. Capper, et al., The nature of telomere fusion and a definition of the critical telomere length in human cells. Genes \& Development 21, 2495-2508 (2007).

34. E. Fragouli, et al., The origin and impact of embryonic aneuploidy. Human Genetics 132, 1001-1013 (2013).

35. E. Fragouli, et al., Cytogenetic analysis of human blastocysts with the use of FISH, CGH and aCGH: scientific data and technical evaluation. Human Reproduction 26, 480-490 (2011).

36. D. Liang, et al., Frequent gene conversion in human embryos induced by double strand breaks. bioRxiv, 2020.06.19.162214 (2020).

37. M. V. Zuccaro, et al., Reading frame restoration at the EYS locus, and allele-specific chromosome removal after Cas9 cleavage in human embryos. bioRxiv, 2020.06.17.149237 (2020).

38. O. Tsaponina, J. E. Haber, Frequent Interchromosomal Template Switches during Gene Conversion in S. cerevisiae. Molecular Cell 55, 615-625 (2014).

39. Y. Li, et al., Patterns of somatic structural variation in human cancer genomes. Nature 578, 112-121 (2020).

40. P. J. Hastings, G. Ira, J. R. Lupski, A Microhomology-Mediated Break-Induced Replication Model for the Origin of Human Copy Number Variation. PLoS Genetics 5, e1000327 (2009).

41. C. Payen, R. Koszul, B. Dujon, G. Fischer, Segmental Duplications Arise from Pol32-Dependent Repair of Broken Forks through Two Alternative Replication-Based Mechanisms. PLoS Genetics 4, e1000175 (2008).

42. T. Gilpatrick, et al., Targeted nanopore sequencing with Cas9-guided adapter ligation. Nature Biotechnology 38, 433-438 (2020).

43. T. Aida, et al., Prime editing primarily induces undesired outcomes in mice. bioRxiv, 2020.08.06.239723 (2020).

44. H. Li, R. Durbin, Fast and accurate long-read alignment with Burrows-Wheeler transform. Bioinformatics 26, 589-595 (2010).

45. I. Scheinin, et al., DNA copy number analysis of fresh and formalin-fixed specimens by shallow wholegenome sequencing with identification and exclusion of problematic regions in the genome assembly. Genome Research 24, 2022-2032 (2014).

46. B. J. Callahan, et al., DADA2: High-resolution sample inference from Illumina amplicon data. Nature Methods 13, 581-583 (2016).

47. L. llie, M. Molnar, RACER: Rapid and accurate correction of errors in reads. Bioinformatics 29, 24902493 (2013).

48. $\quad \mathrm{H}$. Li, et al., The Sequence Alignment/Map format and SAMtools. Bioinformatics 25, 2078-2079 (2009).

49. H. Li, A statistical framework for SNP calling, mutation discovery, association mapping and population genetical parameter estimation from sequencing data. Bioinformatics 27, 2987-2993 (2011).

50. M. I. Love, W. Huber, S. Anders, Moderated estimation of fold change and dispersion for RNA-seq data with DESeq2. Genome Biology 15, 550 (2014). 